\title{
Dominating Sets and Domination Polynomials of Square of Paths
}

\author{
A. Vijayan $^{1}$, K. Lal Gipson ${ }^{2}$ \\ ${ }^{1}$ Department of Mathematics, Nesamony Memorial Christian College, \\ Marthandam, India \\ ${ }^{2}$ Department of Mathematics, Mar Ephraem College of Engineering and Technology, \\ Kanayakumari District, India \\ Email: naacnmccm@gmail.com, lalgipson@yahoo.com
}

Received August 30, 2012; revised September 30, 2012; accepted October 8, 2012

\section{ABSTRACT}

Let $G=(V, E)$ be a simple graph. A set $S \subseteq V$ is a dominating set of $G$, if every vertex in $V$-S is adjacent to at least one vertex in $S$. Let $P_{n}^{2}$ be the square of the Path $P_{n}$ and let $D\left(P_{n}^{2}, i\right)$ denote the family of all dominating sets of $P_{n}^{2}$ with cardinality $i$. Let $d\left(P_{n}^{2}, i\right)=\left|D\left(P_{n}^{2}, i\right)\right|$. In this paper, we obtain a recursive formula for $d\left(P_{n}^{2}, i\right)$. Using this recursive formula, we construct the polynomial, $D\left(P_{n}^{2}, x\right)=\sum_{\left.i=\mid \frac{n}{5}\right]}^{n} d\left(P_{n}^{2}, i\right) x^{i}$, which we call domination polynomial of $P_{n}^{2} \quad$ and obtain some properties of this polynomial.

Keywords: Domination Set; Domination Number; Domination Polynomials

\section{Introduction}

Let $G=(V, E)$ be a simple graph of order $|V|=n$. For any vertex $v \in V$, the open neighbourhood of $v$ is the set $N(v)=\{u \in V / u v \in E\}$ and the closed neighbourhood of $v$ is the set $N[v]=N(v) \cup\{v\}$. For a set $S \subseteq V$, the open neighbourhood of $S$ is $N(S)=U_{n \in S} N(v)$ and the closed neighbourhood of $S$ is $N[S]=N(S) \cup S$. A set $S$ $\subseteq V$ is a dominating set of $G$, if $N[S]=V$, or equivalently, every vertex in $V$-S is adjacent to at least one vertex in $S$. The domination number of a graph $G$ is defined as the minimum size of a dominating set of vertices in $G$ and it is denoted as $\gamma(G)$. A simple path is a path in which all its internal vertices have degree two, and the end vertices have degree one and is denoted by $P_{n}$.

Definition 1.1.: Let $D(G, i)$ be the family of dominating sets of a graph $G$ with Cardinality $i$ and let

$$
d(G, i)=|D(G, i)| .
$$

Then the domination polynomial $D(G, x)$ of $G$ is defined as

$$
D(G, x)=\sum_{i=\gamma(G)}^{|v(G)|} d(G, i) x^{i} .
$$

where $\gamma(G)$ is the domination number of $G$.

Result 1.2. [1]: If a graph $G$ consists of $m$ components
$G_{1}, \cdots, G_{m}$, then

$$
D(G, x)=D\left(G_{1}, x\right) \cdots D\left(G_{m}, x\right) .
$$

Result 1.3. [1]: Let $G_{1}$ and $G_{2}$ be graphs of order $n_{1}$ and $n_{2}$ respectively. Then

$$
\begin{aligned}
D\left(G_{1} \vee G_{2}, x\right) & =\left((1+x)^{n_{1}}-1\right)\left((1+x)^{n_{2}}-1\right) \\
& +D\left(G_{1}, x\right)+D\left(G_{2}, x\right) .
\end{aligned}
$$

Result 1.4. [1]: Let $G$ be a bipartite graph with bipartition $(X, Y)$. Then $G$ contains a matching that saturates every vertex in $\mathrm{X}$ if and only if for all $S \subseteq V|N(S)| \geq|S|$.

Result 1.5. [1]: Let $G$ be a graph of order $n$. Then for every $0 \leq i \leq \frac{n}{2}$, we have $d(G, i) \leq d(G, i+1)$.

Result 1.6. [1]: For any graph $G$ of order $n$, $\gamma\left(G \circ K_{1}\right)=n$.

Result 1.7. [1]: For any graph $G$ of order $n$ and $n \leq m \leq 2 n$, we have

$$
d\left(G \circ K_{1}, m\right)=\left(\begin{array}{c}
n \\
m-n
\end{array}\right) 2^{2 n-m} .
$$

Hence $D\left(G \circ K_{1}, x\right)=x^{n}(x+2)^{n}$.

Definition 1.8: The Square of a graph with the same set of vertices as $G$ and an edge between two vertices if 
and only if there is a path of length at most 2 between them. The second power of a graph is also called its Square.

Let $P_{n}^{2}$ be the square of the path $P_{n}$ (2nd power )with $n$ vertices. Let $D\left(P_{n}^{2}, i\right)$ be the family of dominating sets of the graph $P_{n}^{2}$ with cardinality $i$ and let

$$
d\left(P_{n}^{2}, i\right)=\left|D\left(P_{n}^{2}, i\right)\right| .
$$

We call the polynomial

$$
D\left(P_{n}^{2}, x\right)=\sum_{i=\left\lceil\frac{n}{5}\right\rceil}^{n} d\left(P_{n}^{2}, i\right) x^{i}
$$

the domination polynomial of the graph $P_{n}^{2} \quad$ [2].

In the next section, we construct the families of the dominating sets of the square of paths by recursive method.

As usual we use $\lfloor x\rfloor$ for the largest integer less than or equal to $x$ and $\lceil x\rceil$ for the smallest integer greater than or equal to $x$. Also, we denote the set $\{1,2, \cdots, n\}$ by $[n]$, throughout this paper.

\section{Main Result}

Let $D\left(P_{n}^{2}, i\right)$ be the family of dominating sets of $P_{n}^{2}$ with cardinality $i$. We investigate the dominating sets of $P_{n}^{2}$. We need the following lemma to prove our main results in this section.

Lemma 2.1. [3]: $\gamma\left(P_{n}^{2}\right)=\left\lceil\frac{n}{5}\right\rceil$ By Lemma 2.1 and the definition of domination number, one has the following Lemma:

Lemma 2.2. $D\left(P_{n}^{2}, i\right)=\Phi$ if and only if $i \succ n$ or $i \prec\left\lceil\frac{n}{5}\right\rceil$. A simple path is a path in which all its internal vertices have degree two, and the end vertices have degree one. The following Lemma follows from observation [2].

Lemma 2.3. [2]: If a graph $G$ contains a simple path of length $5 k-1$, then every dominating set of $G$ must contain at least $k$ vertices of the path.

In order to find a dominating set of $P_{n}^{2}$, with cardinality $i$, we only need to consider

$$
\begin{aligned}
& D\left(P_{n-1}^{2}, i-1\right), D\left(P_{n-2}^{2}, i-1\right), \\
& D\left(P_{n-3}^{2}, i-1\right), D\left(P_{n-4}^{2}, i-1\right)
\end{aligned}
$$

and $D\left(P_{n-5}^{2}, i-1\right)$. The families of these dominating sets can be empty or otherwise. Thus, we have eight combinations, whether these five families are empty or not. Two of these combinations are not possible (Lemma 2.5(i), \& (ii)). Also, the combination that

$$
D\left(P_{n-1}^{2}, i-1\right)=D\left(P_{n-2}^{2}, i-1\right)=D\left(P_{n-3}^{2}, i-1\right)=\Phi
$$

$$
D\left(P_{n-4}^{2}, i-1\right)=D\left(P_{n-5}^{2}, i-1\right)=\Phi
$$

does not need to be considered because it implies that $D\left(P_{n}^{2}, i\right)=\Phi \quad$ (See Lemma 2.5 (iii)). Thus we only need to consider five combinations or cases. We consider those cases in Theorem 2.7 .

Lemma 2.4.: If $Y \in D\left(P_{n-6}^{2}, i-1\right)$, and there exists $x$ $\in[n]$ such that $Y \cup\{x\} \in D\left(P_{n}^{2}, i\right)$, then

$$
Y \in D\left(P_{n-5}^{2}, i-1\right) \text {. }
$$

Proof: Suppose that $Y \notin D\left(P_{n-5}^{2}, i-1\right)$. Since

$$
Y \in D\left(P_{n-6}^{2}, i-1\right) \text {, }
$$

$Y$ contains at least one vertex labeled $n-6, n-7$ or $n-8$.

If $n-6 \in Y$, then $Y \in D\left(P_{n-5}^{2}, i-1\right)$, a contradiction. Hence $n-7$ or $n-8 \in Y$, but then in this case, $Y \bigcup\{x\} \notin D\left(P_{n}^{2}, i\right)$, for any $x \in[n]$, also a contradiction.

\section{Lemma 2.5.:}

1) If

$$
D\left(P_{n-1}^{2}, i-1\right)=D\left(P_{n-3}^{2}, i-1\right)=\Phi
$$

then

$$
D\left(P_{n-2}^{2}, i-1\right)=\Phi
$$

2) If

$$
D\left(P_{n-1}^{2}, i-1\right) \neq \Phi, \quad D\left(P_{n-3}^{2}, i-1\right) \neq \Phi
$$

then

$$
D\left(P_{n-2}^{2}, i-1\right) \neq \Phi
$$

3) If

$$
\begin{aligned}
& D\left(P_{n-1}^{2}, i-1\right)=\Phi, \quad D\left(P_{n-2}^{2}, i-1\right)=\Phi, \\
& D\left(P_{n-3}^{2}, i-1\right)=\Phi, \quad D\left(P_{n-4}^{2}, i-1\right)=\Phi, \\
& D\left(P_{n-5}^{2}, i-1\right)=\Phi, \text { then } D\left(P_{n}^{2}, i\right)=\Phi
\end{aligned}
$$

Proof:

1) Since $D\left(P_{n-1}^{2}, i-1\right)=D\left(P_{n-3}^{2}, i-1\right)=\Phi$, by Lemma 2.2, $i-1 \succ n-1$ (or) $i-1 \prec\left\lceil\frac{n-3}{5}\right\rceil$. In either case, we have $D\left(P_{n-2}^{2}, i-1\right)=\Phi$.

2) Suppose that $D\left(P_{n-2}^{2}, i-1\right)=\Phi$, so by Lemma 2.2, we have $i-1 \succ n-2$ or $i-1 \prec\left\lceil\frac{n-2}{5}\right\rceil$ If $i-1 \succ n-2$, then $i-1 \succ n-3$. Therefore $D\left(P_{n-3}^{2}, i-1\right)=\Phi$, a contradiction.

3) Suppose that $D\left(P_{n}^{2}, i\right) \neq \Phi$, Let $Y \in D\left(P_{n}^{2}, i\right)$. Thus, at least one vertex labeled $n$ or $n-1$ or $n-2$ is in $Y$. If $n \in Y$, then by Lemma (2.3), at least one vertex labeled $n-1, n-2, n-3, n-4$ or $n-5$ is in $Y$. If $n-1 \in Y$ or 
$n-2 \in Y$, then $Y-\{n\} \in D\left(P_{n-1}^{2}, i-1\right)$, a contradiction.

If $n-3 \in Y$, then $Y-\{n\} \in D\left(P_{n-2}^{2}, i-1\right)$, a contradiction. Now, suppose that $n-1 \in Y$. Then, by Lemma 2.3 , at least one vertex labeled $n-2, n-3, n-4, n-5$ or $n-6$ is in $Y$. If $n-2 \in Y$ or $n-3 \in Y$, then

$Y-\{n-1\} \in D\left(P_{n-2}^{2}, i-1\right)$ a contradiction. If $n-4 \in Y$, then $Y-\{n-1\} \in D\left(P_{n-3}^{2}, i-1\right)$, a contradiction.

If $n-5 \in Y$, then $Y-\{n-1\} \in D\left(P_{n-4}^{2}, i-1\right)$, a contradiction

If $n-6 \in Y$, then $Y-\{n-1\} \in D\left(P_{n-5}^{2}, i-1\right)$, a contradiction. Therefore, $D\left(P_{n}^{2}, i\right)=\Phi$.

Lemma 2.6.: If $D\left(P_{n}^{2}, i\right) \neq \Phi$, then

1) $D\left(P_{n-1}^{2}, i-1\right)=D\left(P_{n-2}^{2}, i-1\right)=D\left(P_{n-3}^{2}, i-1\right)$ $=D\left(P_{n-4}^{2}, i-1\right)=\Phi$ and $D\left(P_{n-5}^{2}, i-1\right) \neq \Phi$ if and only if $n=5 k$ and $i=k$ for some $k \in N$;

2) $D\left(P_{n-2}^{2}, i-1\right)=D\left(P_{n-3}^{2}, i-1\right)=D\left(P_{n-4}^{2}, i-1\right)$

$=D\left(P_{n-5}^{2}, i-1\right)=\Phi$ then $D\left(P_{n-1}^{2}, i-1\right) \neq \Phi$ if and only if $i=n$;

3) $D\left(P_{n-1}^{2}, i-1\right)=\Phi, D\left(P_{n-2}^{2}, i-1\right) \neq \Phi$, $D\left(P_{n-3}^{2}, i-1\right) \neq \Phi, \quad D\left(P_{n-4}^{2}, i-1\right) \neq \Phi$,

$D\left(P_{n-5}^{2}, i-1\right) \neq \Phi$, if and only if $n=5 k+2$ and $i=\left\lceil\frac{5 k+2}{5}\right\rceil$ for some $k \in N$;

4) $D\left(P_{n-1}^{2}, i-1\right) \neq \Phi, \quad D\left(P_{n-2}^{2}, i-1\right) \neq \Phi$, $D\left(P_{n-3}^{2}, i-1\right) \neq \Phi, \quad D\left(P_{n-4}^{2}, i-1\right) \neq \Phi$, and $D\left(P_{n-5}^{2}, i-1\right)=\Phi$ if and only if $i=n-3$.

5) $D\left(P_{n-1}^{2}, i-1\right) \neq \Phi, \quad D\left(P_{n-2}^{2}, i-1\right) \neq \Phi$, $D\left(P_{n-3}^{2}, i-1\right) \neq \Phi, \quad D\left(P_{n-4}^{2}, i-1\right) \neq \Phi$, and $D\left(P_{n-5}^{2}, i-1\right) \neq \Phi$ if and only if

$$
\left\lceil\frac{n-1}{5}\right\rceil+1 \leq i \leq n-4 \text {. }
$$

\section{Proof:}

1) $(\Rightarrow)$

Since

$$
\begin{aligned}
& D\left(P_{n-1}^{2}, i-1\right)=D\left(P_{n-2}^{2}, i-1\right) \\
& =D\left(P_{n-3}^{2}, i-1\right)=D\left(P_{n-4}^{2}, i-1\right)=\Phi,
\end{aligned}
$$

by Lemma 2.2 ,

$$
i-1 \succ n-1 \text { or } i-1 \prec\left\lceil\frac{n-2}{5}\right\rceil
$$

If $i-1 \succ n-1$, then $i \succ n$ and by Lemma 2.2 ,
$D\left(P_{n}^{2}, i\right)=\Phi$, a contradiction.

So $i \prec\left\lceil\frac{n-2}{5}\right\rceil+1$, and since $D\left(P_{n}^{2}, i\right) \neq \Phi$, we have $\left\lceil\frac{n}{5}\right\rceil \leq i \leq\left\lceil\frac{n-2}{5}\right\rceil+1$, which implies that $n=5 k$ and $i=k$ for some $k \in N$.

$(\Leftarrow)$ If $n=5 k$ and $i=k$ for some $k \in N$, then by Lemma 2.2 ,

$$
\begin{aligned}
& D\left(P_{n-1}^{2}, i-1\right)=D\left(P_{n-2}^{2}, i-1\right) \\
& =D\left(P_{n-3}^{2}, i-1\right)=D\left(P_{n-4}^{2}, i-1\right)=\Phi
\end{aligned}
$$

and $D\left(P_{n-5}^{2}, i-1\right) \neq \Phi$.

2) $(\Rightarrow)$ Since

$$
\begin{aligned}
& D\left(P_{n-2}^{2}, i-1\right)=D\left(P_{n-3}^{2}, i-1\right) \\
& =D\left(P_{n-4}^{2}, i-1\right)=D\left(P_{n-5}^{2}, i-1\right)=\Phi,
\end{aligned}
$$

by Lemma 2.2 ,

$$
i-1 \succ n-2 \text { or } i-1<\left\lceil\frac{n-3}{5}\right\rceil .
$$

If

$$
i-1 \prec\left\lceil\frac{n-3}{5}\right\rceil \text {, then } i-1 \prec\left\lceil\frac{n-1}{5}\right\rceil
$$

and hence $D\left(P_{n-2}^{2}, i-1\right)=\Phi$, a contradiction.

So $i-1 \succ n-2$. Also, $D\left(P_{n-1}^{2}, i-1\right) \neq \Phi$.

Therefore $i \succ n-1$. Therefore $i \geq n$. But $i \leq n$. Hence $i=n$.

$(\Leftarrow)$ If $i=n$, then by lemma 2.2 ,

$$
\begin{aligned}
& D\left(P_{n-2}^{2}, i-1\right)=D\left(P_{n-3}^{2}, i-1\right) \\
& =D\left(P_{n-4}^{2}, i-1\right)=D\left(P_{n-5}^{2}, i-1\right)=\Phi
\end{aligned}
$$

then $D\left(P_{n-1}^{2}, i-1\right) \neq \Phi$.

3) $(\Rightarrow)$ Since $D\left(P_{n-1}^{2}, i-1\right)=\Phi$, by Lemma 2.2,

$i-1 \succ n-1$ or $i-1 \prec\left\lceil\frac{n-1}{5}\right\rceil$.

If $i-1 \succ n-1$, then $i-1 \succ n-2$, by Lemma 2.2 ,

$$
\begin{aligned}
& D\left(P_{n-2}^{2}, i-1\right)=D\left(P_{n-3}^{2}, i-1\right) \\
& =D\left(P_{n-4}^{2}, i-1\right)=D\left(P_{n-5}^{2}, i-1\right)=\Phi,
\end{aligned}
$$

a contradiction.

Therefore $i \prec\left\lceil\frac{n-1}{5}\right\rceil+1$.

But $i-1 \geq\left\lceil\frac{n-2}{5}\right\rceil$, because $D\left(P_{n-2}^{2}, i-1\right) \neq \Phi$.

Therefore $i \geq\left\lceil\frac{n-2}{5}\right\rceil+1$. 
Hence, $\left\lceil\frac{n-2}{5}\right\rceil+1 \leq i \prec\left\lceil\frac{n-1}{5}\right\rceil+1$.

This holds only if $n=5 k+2$ and

for some $k \in N$.

$$
i=k+1=\left\lceil\frac{5 k+2}{5}\right\rceil,
$$

$(\Leftarrow)$ If $n=5 k+2$ and $i=\left\lceil\frac{5 k+2}{5}\right\rceil$ for some $k \in N$, then by Lemma 2.2,

$$
\begin{aligned}
& D\left(P_{n-1}^{2}, i-1\right)=\Phi, \quad D\left(P_{n-2}^{2}, i-1\right) \neq \Phi, \\
& D\left(P_{n-3}^{2}, i-1\right) \neq \Phi, \quad D\left(P_{n-4}^{2}, i-1\right) \neq \Phi
\end{aligned}
$$

and

$$
D\left(P_{n-5}^{2}, i-1\right) \neq \Phi .
$$

4) $\Rightarrow$ Since $D\left(P_{n-5}^{2}, i-1\right)=\Phi$, by Lemma 2.2 ,

$$
i-1 \succ n-5 \text { or } i-1 \prec\left\lceil\frac{n-5}{5}\right\rceil \text {. }
$$

Since $D\left(P_{n-2}^{2}, i-1\right) \neq \Phi$, by Lemma 2.2,

$$
\left\lceil\frac{n-2}{5}\right\rceil+1 \leq i \leq n-1 \text {. }
$$

Therefore $i-1 \prec\left\lceil\frac{n-5}{5}\right\rceil$ is not possible. Therefore $i-1 \succ n-5$. Therefore $i \succ n-4$.

Therefore $i \geq n-3$.

Since $D\left(P_{n-3}^{2}, i-1\right)=\Phi, i-1 \leq n-4$.

Therefore $i \leq n-3$. Hence $i=n-3$.

$(\Leftarrow)$ if $i=n-3$ then

By Lemma 2.2,

$$
\begin{aligned}
& D\left(P_{n-1}^{2}, i-1\right) \neq \Phi, \quad D\left(P_{n-2}^{2}, i-1\right) \neq \Phi, \\
& D\left(P_{n-3}^{2}, i-1\right)=\Phi, \quad D\left(P_{n-4}^{2}, i-1\right) \neq \Phi
\end{aligned}
$$

and

$$
D\left(P_{n-5}^{2}, i-1\right)=\Phi .
$$

5) $(\Rightarrow)$ Since $D\left(P_{n-1}^{2}, i-1\right) \neq \Phi, D\left(P_{n-2}^{2}, i-1\right) \neq \Phi$ $D\left(P_{n-3}^{2}, i-1\right) \neq \Phi$, and $D\left(P_{n-5}^{2}, i-1\right)=\Phi$, then by applying Lemma (2.2),

$$
\begin{aligned}
& \left\lceil\frac{n-1}{5}\right\rceil \leq i-1 \leq n-1,\left\lceil\frac{n-2}{5}\right\rceil \leq i-1 \leq n-2, \\
& \left\lceil\frac{n-3}{5}\right\rceil \leq i-1 \leq n-3,\left\lceil\frac{n-3}{5}\right\rceil \leq i-1 \leq n-4
\end{aligned}
$$

and

$$
\left\lceil\frac{n-3}{5}\right\rceil \leq i-1 \leq n-5
$$

So

$$
\left\lceil\frac{n-1}{5}\right\rceil \leq i-1 \leq n-5
$$

and hence $\left\lceil\frac{n-1}{5}\right\rceil+1 \leq i \leq n-4$.

$(\Leftarrow)$ If $\left\lceil\frac{n-1}{5}\right\rceil+1 \leq i \leq n-4$, then the result follows from Lemma 2.2.

Theorem 2.7.: For every $n \geq 6$ and $i \geq\left\lceil\frac{n}{5}\right\rceil$,

1) If

$$
\begin{aligned}
& D\left(P_{n-1}^{2}, i-1\right)=D\left(P_{n-2}^{2}, i-1\right) \\
& =D\left(P_{n-3}^{2}, i-1\right)=D\left(P_{n-4}^{2}, i-1\right)=\Phi
\end{aligned}
$$

and

$$
D\left(P_{n-5}^{2}, i-1\right) \neq \Phi
$$

then

$$
D\left(P_{n}^{2}, i\right)=\{\{3,8, \cdots, n-7, n-2\}\} .
$$

2) If

$$
\begin{aligned}
& D\left(P_{n-2}^{2}, i-1\right)=D\left(P_{n-3}^{2}, i-1\right) \\
& =D\left(P_{n-4}^{2}, i-1\right)=D\left(P_{n-5}^{2}, i-1\right)=\Phi
\end{aligned}
$$

and

$$
D\left(P_{n-1}^{2}, i-1\right) \neq \Phi
$$

then

$$
D\left(P_{n}^{2}, i\right)=\{[n]\} .
$$

3) If

$$
\begin{aligned}
& D\left(P_{n-1}^{2}, i-1\right)=\Phi, \quad D\left(P_{n-2}^{2}, i-1\right) \neq \Phi, \\
& D\left(P_{n-3}^{2}, i-1\right) \neq \Phi, \quad D\left(P_{n-4}^{2}, i-1\right) \neq \Phi
\end{aligned}
$$

and

$$
D\left(P_{n-5}^{2}, i-1\right) \neq \Phi
$$

then

$$
\begin{aligned}
& D\left(P_{n}^{2}, i\right) \\
& =\left\{\{3,8, \cdots, n-4, n-2\}, X_{1} \cup\{n\}, X_{2} \cup\{n-1\},\right. \\
& X_{3} \cup\{n-2\} / X_{1} \in D\left(P_{n-3}^{2}, i-1\right), \\
& \left.X_{2} \in D\left(P_{n-4}^{2}, i-1\right), X_{3} \in D\left(P_{n-5}^{2}, i-1\right)\right\}
\end{aligned}
$$

4) If $D\left(P_{n-3}^{2}, i-1\right)=\Phi, D\left(P_{n-2}^{2}, i-1\right) \neq \Phi$, $D\left(P_{n-1}^{2}, i-1\right) \neq \Phi$, 
then

5) If

$$
\begin{array}{cc}
D\left(P_{n}^{2}, i\right)=\{[n]-\{x\}, x \in[n]\} . & D\left(P_{n-1}^{2}, i-1\right) \neq \Phi, D\left(P_{n-2}^{2}, i-1\right) \neq \Phi, \\
D\left(P_{n-3}^{2}, i-1\right) \neq \Phi, D\left(P_{n-4}^{2}, i-1\right) \neq \Phi, & D\left(P_{n-5}^{2}, i-1\right) \neq \Phi
\end{array}
$$

then

$$
\begin{aligned}
D\left(P_{n}^{2}, i\right)= & \left\{X_{1} \cup\{n\}, X_{2} \cup\{n-1\}, X_{3} \cup\{n-2\} / X_{1} \in D\left(P_{n-1}^{2}, i-1\right), X_{2} \in D\left(P_{n-2}^{2}, i-1\right), X_{3} \in D\left(P_{n-3}^{2}, i-1\right),\right. \\
& \left.\left\{\{n-2\} \cup\left(X_{4}-X_{3}\right)\right\},\left\{\{n\} \cup\left(X_{4} \cap X_{3}\right)\right\},\left\{\{n-1\} \cup\left(X_{5}-X_{4}\right), / X_{4} \in D\left(P_{n-4}^{2}, i-1\right), X_{5} \in D\left(P_{n-5}^{2}, i-1\right)\right\}\right\}
\end{aligned}
$$

\section{Proof:}

1) Since

$$
\begin{aligned}
& D\left(P_{n-1}^{2}, i-1\right)=D\left(P_{n-2}^{2}, i-1\right) \\
& =D\left(P_{n-3}^{2}, i-1\right)=D\left(P_{n-4}^{2}, i-1\right)=\Phi
\end{aligned}
$$

and $D\left(P_{n-5}^{2}, i-1\right) \neq \Phi$ by Lemma 2.6 i) $n=5 k$, and $i=$ $k$ for some $k \in N$.

Clearly the set $\{3,8, \cdots, n-7, n-2\}$ has $\frac{n}{5}$ elements.

By the definition of $P_{n}^{2}, 3$ has joining with $1,2,4,5$, and 8 has joining with $6,7,9,10$. Therefore 3 and 8 covers all the vertices up to 10 for $n=10$. Proceeding like this, we obtain that $\{3,8, \cdots, n-7, n-2\}$ covers all Vertices up to $n$. The other sets with cardinality $\frac{n}{5}$ are

$\{2,7,12, \cdots, n-3\},\{4,9, \cdots, n-1\}$ etc. In the first set, $n-3$ does not cover the vertex $n$. The second set does not cover the vertex land so on.

Therefore $\{3,8, \cdots, n-7, n-2\}$ is the only dominating set of cardinality $\frac{n}{5}=k=i$.

2) We have

$$
\begin{aligned}
& D\left(P_{n-2}^{2}, i-1\right)=D\left(P_{n-3}^{2}, i-1\right) \\
& =D\left(P_{n-4}^{2}, i-1\right)=D\left(P_{n-5}^{2}, i-1\right)=\Phi
\end{aligned}
$$

and

$$
D\left(P_{n-1}^{2}, i-1\right) \neq \Phi .
$$

By Lemma 2.6 (ii), we have $i=n$. So,

3) We have

$$
D\left(P_{n}^{2}, i\right)=\{[n]\} \text {. }
$$

$$
\begin{aligned}
& D\left(P_{n-1}^{2}, i-1\right)=\Phi, \quad D\left(P_{n-2}^{2}, i-1\right) \neq \Phi, \\
& D\left(P_{n-3}^{2}, i-1\right) \neq \Phi, \quad D\left(P_{n-4}^{2}, i-1\right) \neq \Phi
\end{aligned}
$$

and

$$
D\left(P_{n-5}^{2}, i-1\right) \neq \Phi .
$$

By Lemma 2.6 (iii), $n=5 k+2$ and

$$
i=\left\lceil\frac{5 k+2}{5}\right\rceil=k+1
$$

for some $k \in N$.

Since

$$
\begin{gathered}
X=\{3,8, \cdots, 5 k-2\} \in D\left(P_{5 k}^{2}, k\right), \\
X \bigcup\{5 k+2\} \in D\left(P_{5 k+2}^{2}, k+1\right) .
\end{gathered}
$$

Also, if $X \in D\left(P_{5 k+1}^{2}, k\right)$ then

$$
X \bigcup\{5 k+1\} \in D\left(P_{5 k+2}^{2}, k+1\right)
$$

Therefore, we have

$$
\begin{aligned}
& \left\{\{3,8 \cdots 5 k-2,5 k+1\}, X_{1} \cup\{n\}, X_{2} \cup\{n-1\}, X_{3} \cup\{n-2\} / X_{1} \in D\left(P_{n-3}^{2}, i-1\right),\right. \\
& \left.X_{2} \in D\left(P_{n-4}^{2}, i-1\right), X_{3} \in D\left(P_{n-5}^{2}, i-1\right)\right\} \subseteq D\left(P_{5 k+2}^{2}, k+1\right)
\end{aligned}
$$

Now let $Y \in D\left(P_{5 k+2}^{2}, k+1\right)$. Then $5 k+2$, or $5 k+1$ is in $Y$. If $5 k+2 \in Y$, then by Lemma 3, at least one vertex labeled $5 k+1,5 k$ or $5 k-1$ is in $Y$. If $5 k+1$ or $5 k$ is in $Y$, then

$$
Y-\{5 k+2\} \in D\left(P_{5 k+1}^{2}, k\right),
$$

a contradiction; because $D\left(P_{5 k+1}^{2}, k\right)=\Phi$.

Hence $5 k-1 \in Y, 5 k \notin Y$ and $5 k+1 \notin Y$.

Therefore, $Y=X \bigcup\{5 k+2\}$ for some $X \in D\left(P_{5 k}^{2}, k\right)$; that is $Y=\{3,8, \cdots, 5 k-2,5 k+1\}$. Now suppose that $5 k$ $+1 \in Y$ and $5 k+2 \notin Y$. By Lemma 2.3 at least one vertex labeled $5 k, 5 k-1$ or $5 k-2$ is in $Y$. If $5 k \in Y$ then

$$
Y-\{5 k+1\} \in D\left(P_{5 k}^{2}, k\right)=\{3,8, \cdots, 5 k-2\},
$$

a contradiction because $5 k \notin X$ for all $X \in D\left(P_{5 k}^{2}, k\right)$.

Therefore $5 k-1$, or $5 k-2$ is in $Y$, but $5 k \notin Y$.

Thus $Y=X \bigcup\{5 k+1\}$ for some $X \in D\left(P_{5 k+1}^{2}, k\right)$. So 


$$
\begin{aligned}
& D\left(P_{5 k+2}^{2}, k+1\right) \subseteq\left\{\{3,8, \cdots, 5 k-2,5 k+1\}, X_{1} \cup\{n\}, X_{2} \cup\{n-1\}, X_{3} \cup\{n-2\} / X_{1} \in D\left(P_{n-3}^{2}, i-1\right),\right. \\
& \left.X_{2} \in D\left(P_{n-4}^{2}, i-1\right), X_{3} \in D\left(P_{n-5}^{2}, i-1\right)\right\} \subseteq D\left(P_{5 k+2}^{2}, k+1\right)
\end{aligned}
$$

From (2.3) and (2.4),

$$
\begin{aligned}
& D\left(P_{n}^{2}, i\right)=\{\{3,8, \cdots, n-4, n-2\}, \\
& \left.X_{1} \cup\{n\}, X_{2} \bigcup\{n-1\}, X_{3} \bigcup\{n-2\} / X_{1} \in D\left(P_{n-3}^{2}, i-1\right), X_{2} \in D\left(P_{n-4}^{2}, i-1\right), X_{3} \in D\left(P_{n-5}^{2}, i-1\right)\right\} .
\end{aligned}
$$

4) If

$$
\begin{aligned}
& D\left(P_{n-3}^{2}, i-1\right)=\Phi, \\
& D\left(P_{n-2}^{2}, i-1\right) \neq \Phi, \\
& D\left(P_{n-1}^{2}, i-1\right) \neq \Phi,
\end{aligned}
$$

by Lemma 2.6 (iv) $i=n-1$.

Therefore $D\left(P_{n}^{2}, i\right)=\{[n]-\{x\} \quad x \in[n]\}$.

5) $D\left(P_{n-1}^{2}, i-1\right) \neq \Phi, \quad D\left(P_{n-2}^{2}, i-1\right) \neq \Phi$,

$D\left(P_{n-3}^{2}, i-1\right) \neq \Phi, \quad D\left(P_{n-4}^{2}, i-1\right) \neq \Phi$,

$D\left(P_{n-5}^{2}, i-1\right) \neq \Phi$.

Let $X_{1} \in D\left(P_{n-2}^{2}, i-1\right)$, so at least one vertex labeled $n-1, n-2$ or $n-3$ is in $X_{1}$. If $n-1, n-2$ or $n-3 \in X_{1}$, then

$$
X_{1} \cup\{n\} \in D\left(P_{n}^{2}, i\right) .
$$

Let $X_{2} \in D\left(P_{n-2}^{2}, i-1\right)$, then $n-2$ or $n-3$ or $n-4$ is in $X_{2}$.

If $n-2, n-3$ or $n-4 \in X_{2}$, then

$$
X_{2} \cup\{n-1\} \in D\left(P_{n}^{2}, i\right) \text {. }
$$

Now let $X_{3} \in D\left(P_{n-3}^{2}, i-1\right)$ then $n-3, n-4$ or $n-5$ is in $X_{3}$. If $n-3$ or $n-4$ or $n-5 \in X_{3}$, then

$$
X_{3} \cup\{n-2\} \in D\left(P_{n}^{2}, i\right) \text {. }
$$

Now let $X_{4} \in D\left(P_{n-4}^{2}, i-1\right)$, then $n-4$, or $n-5$ or $n$ -6 is in $X_{4}$. then

If $n-4 \in X_{4}$, then $X_{4} \cup\{n\} \in D\left(P_{n}^{2}, i\right)$. If $n-5 \in X_{4}$,

$$
X_{4} \cup\{n-1\} \in D\left(P_{n}^{2}, i\right) .
$$

If $n-6 \in X_{4}$, then $X_{4} \cup\{n-2\} \in D\left(P_{n}^{2}, i\right)$. Thus we have

$$
\begin{aligned}
& \left\{X_{1} \cup\{n\}, X_{2} \cup\{n-1\}, X_{3} \cup\{n-2\} / X_{1} \in D\left(P_{n-1}^{2}, i-1\right), X_{2} \in D\left(P_{n-2}^{2}, i-1\right), X_{3} \in D\left(P_{n-3}^{2}, i-1\right)\right\} \\
& \cup\left\{\left\{\{n-2\} \cup\left(X_{4}-X_{3}\right)\right\},\left\{\{n\} \cup\left(X_{4} \cap X_{3}\right)\right\},\left\{\{n-1\} \cup\left(X_{5}-X_{4}\right)\right\} / X_{4} \in D\left(P_{n-4}^{2}, i-1\right), X_{5} \in D\left(P_{n-5}^{2}, i-1\right)\right\} \subseteq D\left(P_{n}^{2}, i\right)
\end{aligned}
$$

Now suppose that $n-1 \in Y, n \in Y$ then by Lemma 2.3, at least one vertex labeled $n-2, n-3$ or in $Y$. If $n-2 \epsilon$ $Y$ then $Y=\left(X_{4}-X_{3}\right) \cup\{n-2\}$ for some

$$
X_{4} \in D\left(P_{n-4}^{2}, i-1\right), \quad X_{3} \in D\left(P_{n-3}^{2}, i-1\right) .
$$

Now suppose that, $n-5 \in Y \& n-4 \in Y$, then by

Lemma 2.3 one vertex labeled $n-6, n-7$, in $Y$.

If $n-4 \in Y$, then $Y=\left(X_{5}-X_{4}\right) \cup\{n-1\}$ for

$$
X_{4} \in D\left(P_{n-4}^{2}, i-1\right), \quad X_{5} \in D\left(P_{n-5}^{2}, i-1\right),
$$

So

$$
\begin{aligned}
& D\left(P_{n}^{2}, i\right) \subseteq\left\{X_{1} \cup\{n\}, X_{2} \cup\{n-1\}, X_{3} \cup\{n-2\} / X_{1} \in D\left(P_{n-1}^{2}, i-1\right), X_{2} \in D\left(P_{n-2}^{2}, i-1\right), X_{3} \in D\left(P_{n-3}^{2}, i-1\right)\right\} \\
& \cup\left\{\left\{\{n-2\} \cup\left(X_{4}-X_{3}\right)\right\},\left\{\{n\} \cup\left(X_{4} \cap X_{3}\right)\right\},\left\{\{n-1\} \cup\left(X_{5}-X_{4}\right)\right\} / X_{4} \in D\left(P_{n-4}^{2}, i-1\right), X_{5} \in D\left(P_{n-5}^{2}, i-1\right)\right\}
\end{aligned}
$$

From (2.5) \& (2.6)

$$
\begin{aligned}
& D\left(P_{n}^{2}, i\right)=\left\{X_{1} \cup\{n\}, X_{2} \cup\{n-1\}, X_{3} \cup\{n-2\} / X_{1} \in D\left(P_{n-1}^{2}, i-1\right), X_{2} \in D\left(P_{n-2}^{2}, i-1\right), X_{3} \in D\left(P_{n-3}^{2}, i-1\right)\right\} \\
& \cup\left\{\left\{\{n-2\} \cup\left(X_{4}-X_{3}\right)\right\},\left\{\{n\} \cup\left(X_{4} \cap X_{3}\right)\right\},\left\{\{n-1\} \cup\left(X_{5}-X_{4}\right)\right\} / X_{4} \in D\left(P_{n-4}^{2}, i-1\right), X_{5} \in D\left(P_{n-5}^{2}, i-1\right)\right\}
\end{aligned}
$$

\section{Domination polynomial of $P_{n}^{2}$}

Let $D\left(P_{n}^{2}, x\right)=\sum_{i=\left|\frac{n}{5}\right|}^{n} d\left(P_{n}^{2}, i\right) x^{i}$ be the domination polynomial of a path $P_{n}^{2}$. In this section, we derive the expression for $D\left(P_{n}^{2}, x\right)$.

\section{Theorem 3.1.}

a) If $D\left(P_{n}^{2}, i\right)$ is the family of dominating sets with cardinality i of $P_{n}^{2}$, then 


$$
d\left(P_{n}^{2}, i\right)=d\left(P_{n-1}^{2}, i-1\right)+d\left(P_{n-2}^{2}, i-1\right)+d\left(P_{n-3}^{2}, i-1\right)+d\left(P_{n-4}^{2}, i-1\right)+d\left(P_{n-5}^{2}, i-1\right)
$$

where $d\left(P_{n}^{2}, i\right)=\left|D\left(P_{n}^{2}, i\right)\right|$.

b) For every $n \geq 6$,

$$
D\left(P_{n}^{2}, x\right)=x\left[D\left(P_{n-1}^{2}, x\right)+D\left(P_{n-2}^{2}, x\right)+D\left(P_{n-3}^{2}, x\right)+D\left(P_{n-4}^{2}, x\right)+D\left(P_{n-5}^{2}, x\right)\right]
$$

with the initial values

$$
\begin{aligned}
& D\left(P_{1}^{2}, x\right)=x, D\left(P_{2}^{2}, x\right)=x^{2}+2 x, \\
& D\left(P_{3}^{2}, x\right)=x^{3}+3 x^{2}+3 x, \\
& D\left(P_{4}^{2}, x\right)=x^{4}+4 x^{3}+6 x^{2}+2 x, \\
& D\left(P_{5}^{2}, x\right)=x^{5}+5 x^{4}+10 x^{3}+8 x^{2}+x .
\end{aligned}
$$

\section{Proof:}

a) Using (i), (ii), (iii), (iv) and (v) of Theorem 2.7, we prove (a) part.

1) Suppose (i) of Theorem 2.7 holds.

From (v), we have

$$
D\left(P_{n}^{2}, i\right)=\left\{\Phi \bigcup\{n-1\} \bigcup\left(X_{5}\right) X_{5} \in D\left(P_{n-5}^{2}, i-1\right)\right\} .
$$

Since

$$
\begin{gathered}
D\left(P_{n-1}^{2}, i-1\right)=D\left(P_{n-2}^{2}, i-1\right)=\Phi, \\
D\left(P_{n-3}^{2}, i-1\right)=D\left(P_{n-4}^{2}, i-1\right)=\Phi \\
\left|D\left(P_{n-1}^{2}, i-1\right)\right|=\left|D\left(P_{n-2}^{2}, i-1\right)\right|=0
\end{gathered}
$$

$$
\left|D\left(P_{n-3}^{2}, i-1\right)\right|=\left|D\left(P_{n-4}^{2}, i-1\right)\right|=0
$$

Therefore $\left|D\left(P_{n}^{2}, i\right)\right|=\left|D\left(P_{n-5}^{2}, i-1\right)\right|$.

Therefore $d\left(P_{n}^{2}, i\right)=d\left(P_{n-5}^{2}, i-1\right)$.

Therefore, in this case $d\left(P_{n}^{2}, i\right)=d\left(P_{n-5}^{2}, i-1\right)$ holds.

2) Suppose (ii) of Theorem 2.7 holds.

From (v), we have

$$
D\left(P_{n}^{2}, i\right)=\left\{\Phi \bigcup\{n\} \bigcup\left(X_{1}\right) X_{1} \in D\left(P_{n-1}^{2}, i-1\right)\right\} .
$$

Since $D\left(P_{n-1}^{2}, i-1\right)=D\left(P_{n-3}^{2}, i-1\right)=\Phi$

$$
\begin{aligned}
& D\left(P_{n-4}^{2}, i-1\right)=D\left(P_{n-5}^{2}, i-1\right)=\Phi \\
& \left|D\left(P_{n-2}^{2}, i-1\right)\right|=\left|D\left(P_{n-3}^{2}, i-1\right)\right|=0 \\
& \left|D\left(P_{n-4}^{2}, i-1\right)\right|=\left|D\left(P_{n-5}^{2}, i-1\right)\right|=0
\end{aligned}
$$

Therefore $\left|D\left(P_{n}^{2}, i\right)\right|=\left|D\left(P_{n-1}^{2}, i-1\right)\right|$

Therefore $d\left(P_{n}^{2}, i\right)=d\left(P_{n-1}^{2}, i-1\right)$.

Therefore, In this case $d\left(P_{n}^{2}, i\right)=d\left(P_{n-1}^{2}, i-1\right)$ holds.

3) Suppose (iii) of Theorem 2.7 holds.

From (v), we have

$$
\begin{aligned}
& D\left(P_{n}^{2}, i\right)=\left\{\Phi \bigcup\{n-1\} \cup\left(X_{2}\right),\{n-2\} \cup\left(X_{3}\right), \cup\left(X_{4}-X_{3}\right) \cup\{n-2\},\left(X_{4} \cap X_{3}\right) \cup\{n\},\right. \\
& \left.\left(X_{5}-X_{4}\right) \cup\{n-1\} / X_{2} \in D\left(P_{n-2}^{2}, i-1\right), X_{3} \in D\left(P_{n-3}^{2}, i-1\right), X_{4} \in D\left(P_{n-4}^{2}, i-1\right) \text { and } X_{5} \in D\left(P_{n-5}^{2}, i-1\right)\right\}
\end{aligned} .
$$

Since

$$
D\left(P_{n-1}^{2}, i-1\right)=\Phi,\left|D\left(P_{n-1}^{2}, i-1\right)\right|=0 .
$$

Therefore

$$
\begin{aligned}
\left|D\left(P_{n}^{2}, i\right)\right|=\left|D\left(P_{n-2}^{2}, i-1\right)\right| \cup\left|D\left(P_{n-3}^{2}, i-1\right)\right| & +d\left(P_{n-5}^{2}, i-1\right) . \\
\cup\left|D\left(P_{n-4}^{2}, i-1\right)\right| \cup\left|D\left(P_{n-5}^{2}, i-1\right)\right| & \text { 4) Suppose (v) of Theorem 2.7 holds. } \\
D\left(P_{n}^{2}, i\right)= & \left\{\{n\} \cup\left(X_{1}\right),\{n-1\} \cup\left(X_{2}\right),\{n-2\} \cup\left(X_{3}\right), \cup\left(X_{4}-X_{3}\right) \cup\{n-2\},\left(X_{4} \cap X_{3}\right) \cup\{n\},\right. \\
& \left(X_{5}-X_{4}\right) \cup\{n-1\} / X_{1} \in D\left(P_{n-1}^{2}, i-1\right), X_{2} \in D\left(P_{n-2}^{2}, i-1\right), X_{3} \in D\left(P_{n-3}^{2}, i-1\right), \\
& \left.X_{4} \in D\left(P_{n-4}^{2}, i-1\right) \text { and } X_{5} \in D\left(P_{n-5}^{2}, i-1\right)\right\} .
\end{aligned}
$$

Therefore 


$$
\left|D\left(P_{n}^{2}, i\right)\right|=\left|D\left(P_{n-1}^{2}, i-1\right)\right| \cup\left|D\left(P_{n-2}^{2}, i-1\right)\right| \cup\left|D\left(P_{n-3}^{2}, i-1\right)\right| \cup\left|D\left(P_{n-4}^{2}, i-1\right)\right| \cup\left|D\left(P_{n-5}^{2}, i-1\right)\right| .
$$

Therefore

$$
\sum d\left(P_{n}^{2}, i\right)=d\left(P_{n-1}^{2}, i-1\right)+d\left(P_{n-2}^{2}, i-1\right)+d\left(P_{n-3}^{2}, i-1\right)+d\left(P_{n-4}^{2}, i-1\right)+d\left(P_{n-5}^{2}, i-1\right) .
$$

Therefore, we have the theorem.

b)

$$
\begin{aligned}
d\left(P_{n}^{2}, i\right) x^{i}= & d\left(P_{n-1}^{2}, i-1\right) x^{i}+d\left(P_{n-2}^{2}, i-1\right) x^{i}+d\left(P_{n-3}^{2}, i-1\right) x^{i}+d\left(P_{n-4}^{2}, i-1\right) x^{i}+d\left(P_{n-5}^{2}, i-1\right) x^{i} \\
\sum d\left(P_{n}^{2}, i\right) x^{i} & =\sum d\left(P_{n-1}^{2}, i-1\right) x^{i}+\sum d\left(P_{n-2}^{2}, i-1\right) x^{i}+\sum d\left(P_{n-3}^{2}, i-1\right) x^{i}+\sum d\left(P_{n-4}^{2}, i-1\right) x^{i}+\sum d\left(P_{n-5}^{2}, i-1\right) x^{i} \\
d\left(P_{n}^{2}, i\right) x^{i}= & x \sum d\left(P_{n-1}^{2}, i-1\right) x^{i-1}+x \sum d\left(P_{n-2}^{2}, i-1\right) x^{i-1}+x \sum d\left(P_{n-3}^{2}, i-1\right) x^{i-1} \\
+ & x \sum d\left(P_{n-4}^{2}, i-1\right) x^{i-1}+x \sum d\left(P_{n-5}^{2}, i-1\right) x^{i-1} \\
\sum d\left(P_{n}^{2}, i\right) x^{i}= & x\left[\sum d\left(P_{n-1}^{2}, i-1\right) x^{i-1}+\sum d\left(P_{n-2}^{2}, i-1\right) x^{i-1}+\sum d\left(P_{n-3}^{2}, i-1\right) x^{i-1}\right. \\
& \left.+\sum d\left(P_{n-4}^{2}, i-1\right) x^{i-1}+\sum d\left(P_{n-5}^{2}, i-1\right) x^{i-1}\right] \\
D\left(P_{n}^{2}, x\right)=x & {\left[D\left(P_{n-1}^{2}, x\right)+D\left(P_{n-2}^{2}, x\right)+D\left(P_{n-3}^{2}, x\right)+D\left(P_{n-4}^{2}, x\right)+D\left(P_{n-5}^{2}, x\right)\right] }
\end{aligned}
$$

with the initial values

$$
\begin{aligned}
& D\left(P_{1}^{2}, x\right)=x, D\left(P_{2}^{2}, x\right)=x^{2}+2 x, \\
& D\left(P_{3}^{2}, x\right)=x^{3}+3 x^{2}+3 x \\
& D\left(P_{4}^{2}, x\right)=x^{4}+4 x^{3}+6 x^{2}+2 x, \\
& D\left(P_{5}^{2}, x\right)=x^{5}+5 x^{4}+10 x^{3}+8 x^{2}+x .
\end{aligned}
$$
1.

We obtain $d\left(P_{n}^{2}, i\right)$ for $1 \leq n \leq 10$ as shown in Table

Table 1. $d\left(P_{n}^{2}, i\right)$, the number of dominating set of $P_{n}^{2}$ with cardinality $i$.

\begin{tabular}{ccccccccccc}
\hline$i$ & 1 & 2 & 3 & 4 & 5 & 6 & 7 & 8 & 9 & 10 \\
\hline$n$ & & & & & & & & & & \\
1 & 1 & & & & & & & & & \\
2 & 2 & 1 & & & & & & & & \\
3 & 3 & 3 & 1 & & & & & & & \\
4 & 2 & 6 & 4 & 1 & & & & & & \\
5 & 1 & 8 & 10 & 5 & 1 & & & & & \\
6 & 0 & 9 & 18 & 15 & 6 & 1 & & & & \\
7 & 0 & 8 & 27 & 33 & 21 & 7 & 1 & & & \\
8 & 0 & 6 & 34 & 60 & 54 & 28 & 8 & 1 & & \\
9 & 0 & 3 & 37 & 93 & 114 & 82 & 36 & 9 & 1 & \\
10 & 0 & 1 & 34 & 126 & 206 & 196 & 118 & 45 & 10 & 1 \\
\hline
\end{tabular}

In the following Theorem, we obtain some properties of $d\left(P_{n}^{2}, i\right)$

Theorem 3.2. The following properties hold for the coefficients of $D\left(P_{n}^{2}, x\right)$;

1) $d\left(P_{5 n}^{2}, n\right)=1$ for every $n \in N$.

2) $d\left(P_{n}^{2}, n\right)=1$ for every $n \in N$

3) $d\left(P_{n}^{2}, n-1\right)=n$ for every $n \geq 2$

4) $d\left(P_{n}^{2}, n-2\right)=\frac{n(n-1)}{2}$ for every $n \geq 3$

5) $d\left(P_{n}^{2}, n-3\right)=n C_{3}-2$ for every $n \geq 4$

6) $d\left(P_{n}^{2}, n-4\right)=n C_{4}-(2 n-6)$ for every $n \geq 5$

7) $d\left(P_{5 n-1}^{2}, n\right)=n+1$ for every $n \in N$.

\section{Proof:}

1) Since $D\left(P_{5 n}^{2}, n\right)=\{3,8, \cdots, 5 k-2\}$, we have $d\left(P_{5 n}^{2}, n\right)=1$.

2) Since $D\left(P_{n}^{2}, n\right)=\{[n]\}$, we have the result

3) Since $D\left(P_{n}^{2}, n-1\right)=\{[n]-\{x\} x \in[n]\}$, we have $d\left(P_{n}^{2}, n-1\right)=n$.

4) By induction on $n$. The result is true for $n=3$.

L.H.S. $=d\left(P_{3}^{2}, 1\right)=3 \quad$ (from table)

$$
\text { R.H.S }=\left(\frac{3 \times 2}{2}\right)=3
$$

Therefore, the result is true for $n=4$.

Now suppose that the result is true for all numbers less than " $n$ " and we prove it for $n$.

By Theorem 3.1, 


$$
\begin{aligned}
& d\left(P_{n}^{2}, n-2\right)=d\left(P_{n-1}^{2}, n-3\right)+d\left(P_{n-2}^{2}, n-3\right) \\
& +d\left(P_{n-3}^{2}, n-3\right)+d\left(P_{n-4}^{2}, n-3\right)+d\left(P_{n-5}^{2}, n-3\right) \\
& =\frac{(n-1)(n-2)}{2}+(n-2)+1 \\
& =\frac{n^{2}-3 n+2+2 n-4+2}{2}=\frac{n^{2}-n}{2}=\frac{n(n-1)}{2} \text {. } \\
& \text { 5) By induction on } n \text {. The result is true for } n=4 \text {. } \\
& \text { L.H.S }=d\left(P_{4}^{2}, 1\right)=2 \text { (from table) } \\
& \text { R.H.S }=d\left(P_{4}^{2}, 1\right)=\frac{4 \times 3 \times 2}{6}-2 \\
& d\left(P_{n}^{2}, n-3\right)=d\left(P_{n-1}^{2}, n-4\right)+d\left(P_{n-2}^{2}, n-4\right)+d\left(P_{n-3}^{2}, n-4\right)+d\left(P_{n-4}^{2}, n-4\right)+d\left(P_{n-5}^{2}, n-4\right) \\
& =\frac{(n-1)(n-2)(n-3)}{6}-2+\frac{(n-1)(n-2)}{2}+(n-3)+1 \\
& =\frac{1}{6}[(n-1)(n-2)(n-3)+3(n-2)(n-3)+6(n-3)-12+6] \\
& =\frac{1}{6}[(n-3)[(n-2)(n+2)+6]-6]=\frac{1}{6}\left[(n-3)\left[n^{2}-4+6\right]-6\right] \\
& =\frac{1}{6}\left[n^{3}+2 n-3 n^{2}-6-6\right]=\frac{1}{6}\left[n^{3}-3 n^{2}+2 n-12\right]
\end{aligned}
$$

Therefore the result is true for $n=1$. Now suppose the

6) By induction on $n$. Let $n=5$

L.H.S $=d\left(P_{5}^{2}, 1\right)=1 \quad($ from table $)$

$$
\text { R.H.S }=\frac{n(n-1)(n-2)(n-3)}{1 \times 2 \times 3 \times 4}-(2 n-6)=\frac{5 \times 4 \times 3 \times 2}{1 \times 2 \times 3 \times 4}-(2 \times 5-6)=5-4=1
$$

Therefore the result is true for $n=1$.

Now suppose that the result is true for all natural numbers less than or equal to $n$.

$$
\begin{aligned}
& d\left(P_{n}^{2}, n-4\right)=d\left(P_{n-1}^{2}, n-5\right)+d\left(P_{n-2}^{2}, n-5\right)+d\left(P_{n-3}^{2}, n-5\right)+d\left(P_{n-4}^{2}, n-5\right)+d\left(P_{n-5}^{2}, n-5\right) \\
& =\frac{n(n-1)(n-2)(n-3)(n-4)}{24}(2(n-1)-6)+\frac{(n-2)(n-3)(n-4)}{6} 2+\frac{(n-3)(n-4)}{2}+(n-4)+1 \\
& =\frac{1}{24}[(n-1)(n-2)(n-3)(n-4)-12(n-2)(n-3)(n-4)+24(n-4)+4(n-2)(n-3)(n-4)+48 n+192-48+24] \\
& =\frac{1}{24}[(n-2)(n-3)(n-4)[n-1+4]+12(n-4)[n-3+2]-48 n+168] \\
& =\frac{1}{24}[(n-2)(n-3)(n-4)(n+3)+12(n-4)(n-1)-48 n+168] \\
& =\frac{1}{24}[(n-4)[(n-2)(n-3)(n+3)+12(n-1)-48 n+168]] \\
& =\frac{1}{24}\left[(n-4)\left[(n-2)\left(n^{2}-9\right)+12 n-12-48 n\right]\right]=\frac{1}{24}\left[(n-4)\left(n^{3}-9 n-2 n^{2}+18\right)-36 n+156\right] \\
& =\frac{1}{24}\left[n^{4}-9 n^{2}-2 n^{3}+18 n-4 n^{3}+36 n+8 n^{2}+72-36 n+156\right]=\frac{1}{24}\left[n^{4}-6 n^{3}+11 n^{2}-54 n+144\right]
\end{aligned}
$$

7) From the table it is true.

Theorem 3.3.

$$
d\left(P_{n+1}^{2}, j+1\right)-d\left(P_{n}^{2}, j+1\right)=d\left(P_{n}^{2}, j\right)-d\left(P_{n-5}^{2}, j\right)
$$

1) $\sum_{i=n}^{5 n} d\left(P_{i}^{2}, n\right)=5 \sum_{i=2}^{5 n-5} d\left(P_{i}^{2}, n-1\right)$

2) For every $j \geq\left\lceil\frac{n}{5}\right\rceil$,

3) If $S_{n}=\sum_{\left.i=\mid \frac{n}{5}\right\rceil}^{n} d\left(P_{n}^{2}, j\right)$, then for every $n \geq 6$, $S_{n}=S_{n-1}+S_{n-2}+S_{n-3}+S_{n-4}+S_{n-5}$ with initial values $S_{1}$ 
$=1, S_{2}=3, S_{3}=7, S_{4}=13$ and $S_{5}=25$.

Proof:
1) Proof by induction on $n$

First suppose that $n=2$ then,

$\sum_{i}^{10} d\left(P_{i}^{2}, 2\right)=45=5 \sum_{i=2}^{5} d\left(P_{i}^{2}, 1\right)$

$\sum_{i=k}^{5 k} d\left(P_{i}^{2}, k\right)=\sum_{i=k}^{5 k} d\left(P_{i-1}^{2}, k-1\right)+\sum_{i=k}^{5 k} d\left(P_{i-2}^{2}, k-1\right)+\sum_{i=k}^{5 k} d\left(P_{i-3}^{2}, k-1\right)+\sum_{i=k}^{5 k} d\left(P_{i-4}^{2}, k-1\right)+\sum_{i=k}^{5 k} d\left(P_{i-5}^{2}, k-1\right)$

$=5 \sum_{i=k-1}^{5(k-1)} d\left(P_{i-1}^{2}, k-2\right)+5 \sum_{i=k-1}^{5(k-1)} d\left(P_{i-2}^{2}, k-2\right)+5 \sum_{i=k-1}^{5(k-1)} d\left(P_{i-3}^{2}, k-2\right)+5 \sum_{i=k-1}^{5(k-1)} d\left(P_{i-4}^{2}, k-2\right)+5 \sum_{i=k-1}^{5(k-1)} d\left(P_{i-5}^{2}, k-2\right)$

$=5 \sum_{i=k-1}^{5 k-5} d\left(P_{i}^{2}, k-1\right)$

We have the result.

2) By Theorem 3.1, We have

$$
\begin{aligned}
d\left(P_{n+1}^{2}, j+1\right)-d\left(P_{n}^{2}, j+1\right) & =d\left(P_{n}^{2}, j\right)+d\left(P_{n-1}^{2}, j\right)+d\left(P_{n-2}^{2}, j\right)+d\left(P_{n-3}^{2}, j\right) \\
& +d\left(P_{n-4}^{2}, j\right)+d\left(P_{n-1}^{2}, j\right)-d\left(P_{n-1}^{2}, j\right)-d\left(P_{n-2}^{2}, j\right)-d\left(P_{n-3}^{2}, j\right)-d\left(P_{n-4}^{2}, j\right)-d\left(P_{n-5}^{2}, j\right) \\
d\left(P_{n+1}^{2}, j+1\right)-d\left(P_{n}^{2}, j+1\right) & =d\left(P_{n}^{2}, j\right)-d\left(P_{n-5}^{2}, j\right)
\end{aligned}
$$

Therefore we have the result

3) By theorem (3.1), we have

$$
\begin{aligned}
S_{n} & =\sum_{i=\left\lceil\frac{n}{5}\right\rceil}^{n} d\left(P_{n}^{2}, j\right) \\
S_{n} & =\sum_{i=\left\lceil\frac{n}{5}\right\rceil}^{n}\left[d\left(P_{n-1}^{2}, j-1\right)+d\left(P_{n-2}^{2}, j-1\right)+d\left(P_{n-3}^{2}, j-1\right)+d\left(P_{n-4}^{2}, j-1\right)+d\left(P_{n-5}^{2}, j-1\right)\right] \\
& =\sum_{\left.i=\mid \frac{n}{5}\right\rceil-1}^{n-1} d\left(P_{n-1}^{2}, j-1\right)+\sum_{\left.i=\mid \frac{n}{5}\right]-1}^{n-1} d\left(P_{n-2}^{2}, j-1\right)+\sum_{\left.i=\mid \frac{n}{5}\right\rceil-1}^{n-1} d\left(P_{n-3}^{2}, j-1\right)+\sum_{\left.i=\mid \frac{n}{5}\right]-1}^{n-1} d\left(P_{n-4}^{2}, j-1\right)+\sum_{\left.i=\mid \frac{n}{5}\right\rceil-1}^{n-1} d\left(P_{n-5}^{2}, j-1\right) \\
S_{n} & =S_{n-1}+S_{n-2}+S_{n-3}+S_{n-4}+S_{n-5} .
\end{aligned}
$$

\section{Conclusions}

In [2], the domination polynomial of path was studied and obtained the very important property,

$$
d\left(P_{n}^{2}, i\right)=d\left(P_{n-1}^{2}, i-1\right)+d\left(P_{n-2}^{2}, i-1\right)+d\left(P_{n-3}^{2}, i-1\right) .
$$

It is interesting that we have derived an analogues relation for the square of path of the form

$$
\begin{aligned}
d\left(P_{n}^{2}, i\right)= & d\left(P_{n-1}^{2}, i-1\right)+d\left(P_{n-2}^{2}, i-1\right)+d\left(P_{n-3}^{2}, i-1\right) \\
& +d\left(P_{n-4}^{2}, i-1\right)+d\left(P_{n-5}^{2}, i-1\right)
\end{aligned}
$$

One can characterise the roots of the polynomial $D\left(P_{n}^{2}, x\right)$ and identify whether they are real or complex.
Another interesting character $\mathrm{r}$ to be investigated is whether $D\left(P_{n}^{2}, x\right)$ is log concave are not.

\section{REFERENCES}

[1] S. Alikhani and Y.-H. Peng, "Introduction to Domination Polynomial of a Graph,” .arXiv:0905.2251v1[math.co], 2009.

[2] S. Alikhani and Y.-H. Peng, "Domination Sets and Domination Polynomials of Paths," International Journal of Mathematics and Mathematical Sciences, Vol. 2009, 2009, Article ID: 542040.

[3] G. Chartand and P. Zhang, "Introduction to Graph Theory," McGraw-Hill, Boston, 2005. 